\title{
Poloidal Asymmetry of Parallel Rotation Measured in ASDEX Upgrade
}

\author{
T Pütterich, E Viezzer, R Dux, RM McDermott and the ASDEX \\ Upgrade Team \\ Max-Planck-Institut für Plasmaphysik, EURATOM Association, Boltzmannstraße 2, \\ D-85748 Garching, Germany
}

\begin{abstract}
The parallel flows in the H-mode edge of ASDEX Upgrade are investigated. Beam-based charge-exchange recombination spectroscopy (CXRS) provides the toroidal and poloidal impurity flow velocities at the outboard midplane, while a deuterium-puff based CXRS measurement provides the toroidal impurity flow velocities at the inboard midplane. In order to more easily compare these measurements to fundamental boundary conditions, a basic overview of flows on a flux surface is presented. The boundary conditions are given by the continuity equation and mean that the flow velocities on a flux surface must have a specific structure in order to provide zero divergence. At first, poloidal impurity density asymmetries and radial transport are neglected. Inside of the pedestal-top of the electron density profile the measurements agree with the postulated flow structure, while they do not agree at the pedestal itself. Here, an extension of the theoretical scheme, which allows for a poloidal impurity density asymmetry, suggests that the measured flow velocities could be explained by an excess impurity density at the inboard midplane. In detail, the inboard impurity density is postulated to be at the separatrix up to a factor of 6.5 higher than impurity density at the outboard midplane. Near the pedestal-top of the electron density, this asymmetry disappears. Radial transport is considered as an explanation for that asymmetry. A conclusive disentanglement of the driving mechanisms requires further investigation.
\end{abstract}

\section{Nuclear Fusion}

E-mail: Thomas.Puetterich@ipp.mpg.de

\section{Introduction}

The flows in the plasma edge of a tokamak fusion plasma have a complicated structure and at the same time they are an important ingredient of the physics that rules the transport at the plasma edge. For instance, the shear of the plasma flows perpendicular to the magnetic field lines is closely connected to an edge transport barrier in high confinement discharges (H-modes) [1]. The corresponding steep gradients in densities and temperatures are selfconsistent with the flows and thus the radial electric field. However, the parallel flows are little investigated. One basic boundary condition, which they have to meet is given by the continuity equation, which for steady state describes the total flow $\vec{v}$, by $\vec{\nabla} \cdot(n \vec{v})=0$, where $\mathrm{n}$ is the particle density. If radial transport is assumed to be poloidally symmetric, i.e. divergence-free, the perpendicular velocity is closely connected to the parallel velocity via the above equation.

When investigating the H-mode edge at ASDEX Upgrade [2], the measurements from charge exchange recombination spectroscopy (CXRS) gave unexpected rotation profiles in between the occurrence of two edge-localized modes (ELMs). A local minimum in the parallel flow at the radius of the electron density pedestal-top was observed. In Ref. [3] and 
in the present work, these profiles are presented. Since high-viscosity and strong magnetic braking of the plasma is expected only at the separatrix, the local minimum in the rotation is quite puzzling. Instead of postulating complicated transport or torque profiles, it is attempted to explain these profiles by shedding light on the poloidal asymmetry of the parallel flows. The measurements in Ref. [3] are performed on the outboard midplane and it is possible that they are not representative of the full flux surface. Such poloidal asymmetries are evaluated in the present work by applying the continuity equation to the flow measurements on a flux surface. This work is closely connected to the work in Ref. [4] performed at Alcator CMod. This paper is organized as follows: In section 2, the basics of the flow structure on an isolated flux surface are presented. In section 3 the extent to which this structure is visible in experiment and how the diagnostic measurements can be compared to the theoretical scheme are described. In section 4 the diagnostics are introduced in more detail. In section 5 the experiment and the results are presented, while in section 6 they are discussed and compared to the expected flow structure as described in section 3 . In section 7 , the discussion is extended to a scheme in which the impurity densities are not constant on each flux surface. Finally, in section 8 the effects of radial transport and its non-zero divergence are discussed. Section 9 is the summary.

\section{Flow Structure on a Flux Surface}

The following considerations are basic and have been presented in review papers (e.g. Ref. [5]). The equations are rewritten and reconsidered in this work, to clarify exactly to which terms the experimental data is compared.

The general continuity equation requires that the 3D flow velocity exhibits zero divergence. In detail, it is required that $\vec{\nabla} \cdot\left(n_{\alpha} \vec{v}_{\alpha}\right)=0$, where $n_{\alpha}$ is the density of a species $\alpha$ and $\vec{v}_{\alpha}$ its flow velocity. When the divergence of radial flows is negligible compared to the fluxes within a flux surface, the $2 \mathrm{D}$ flows on a flux surface must be divergence free. In sections 2 to 7, the radial divergence of flows is assumed to be zero and the structure of the 2D flows on a flux surface are investigated, while the perpendicular flow velocities are obtained by the radial force balance.

The radial force balance (Eq. 1 ) for an ion species $\alpha$ relates the local velocity $\vec{v}_{\alpha}$ to the local magnetic field $\vec{B}$, electric field $\vec{E}$ and the diamagnetic term $-\frac{\vec{\nabla} p_{\alpha}}{Z_{\alpha} e n_{\alpha}}$, where $p_{\alpha}$ is the pressure, $Z_{\alpha}$ the charge, $n_{\alpha}$ the density of the species $\alpha$ and $e$ the elementary charge. The radial force balance is given by

$$
0=\vec{E}-\frac{\vec{\nabla} p_{\alpha}}{Z_{\alpha} e n_{\alpha}}+\vec{v}_{\alpha} \times \vec{B} \Rightarrow v_{\perp, \alpha}=\left(E_{r}-\frac{1}{Z_{\alpha} e n_{\alpha}} \frac{\partial p_{\alpha}}{\partial r}\right) \frac{1}{B}
$$

where $r$ is the radial coordinate, $E_{r}$ the radial electric field, $v_{\perp, \alpha}$ the velocity component perpendicular within the flux surface to $\vec{B}$ and $B \vec{e}_{\|}=\vec{B}$, where $\vec{e}_{\|}$is the unity vector along a magnetic field line. The two contributions to $v_{\perp, \alpha}$ are the $\vec{E} x \vec{B}$ velocity and the diamagentic drift velocity. It should be noted that $v_{\perp, \alpha}$ is different for the inner (high field side, HFS) and outer (low field side, LFS) midplane, which is the basic reason for non-zero divergence of $v_{\perp, \alpha}$ on a flux surface. This is compensated for by the parallel Pfirsch-Schlüter flows, a 
derivation of which is presented in the following. Equation 1 may be rewritten as

$$
\begin{aligned}
v_{\perp, \alpha} \vec{e}_{\perp} & =-\frac{\vec{\nabla} \Phi \times \vec{B}}{B^{2}}-\frac{1}{Z_{\alpha} e n_{\alpha}} \frac{\vec{\nabla} p_{\alpha} \times \vec{B}}{B^{2}} \\
& =-\left(\frac{\partial \Phi}{\partial \Psi}+\frac{1}{Z_{\alpha} e n_{\alpha}} \frac{\partial p_{\alpha}}{\partial \Psi}\right) \frac{\vec{\nabla} \Psi \times \vec{B}}{B^{2}}=-\omega_{\alpha}(\Psi) \frac{\vec{\nabla} \Psi \times \vec{B}}{B^{2}}
\end{aligned}
$$

with $\omega_{\alpha}(\Psi)=\frac{\partial \Phi}{\partial \Psi}+\frac{1}{Z_{\alpha} e n_{\alpha}} \frac{\partial p_{\alpha}}{\partial \Psi}$, where $\Phi$ is the electrostatic potential, $\Psi$ is the poloidal magnetic flux and $\vec{e}_{\perp}$ is the local unity vector tangential to the flux surface and perpendicular to the magnetic field line. The last equation assumes $\Phi, n_{\alpha}$ and $p_{\alpha}$ to be constant on the flux surface such that $\omega_{\alpha}$ is a pure flux function. Note that $\omega_{\alpha}(\Psi)$ is identified below as the angular frequency of a toroidal rigid body rotation. Using the equality $\frac{\vec{\nabla} \Psi \times \vec{B}}{B^{2}}=\frac{R B_{p}}{B} \vec{e}_{\perp}$ valid for axisymmetric systems, where $B_{p}$ is the poloidal magnetic field component and $R$ is the local major radius, Eq. 2 becomes:

$$
v_{\perp, \alpha} \vec{e}_{\perp}=-\omega_{\alpha}(\Psi) \frac{R B_{p}}{B} \vec{e}_{\perp}
$$

This can be further manipulated using relations between the unity vectors for the toroidal $\left(\vec{e}_{t}\right)$, poloidal $\left(\vec{e}_{p}\right)$, parallel $\left(\vec{e}_{\|}\right)$and perpendicular $\left(\vec{e}_{\perp}\right)$ directions, while the same indices on $B$ indicate the corresponding components of the magnetic field. Note that the unity vector along the plasma radius $\vec{e}_{r}, \vec{e}_{\|}$and $\vec{e}_{\perp}$ are chosen such that they form a right-handed system. The equalities $\vec{e}_{\perp}=\frac{B_{t}}{B} \vec{e}_{p}-\frac{B_{p}}{B} \vec{e}_{t}$ and $\vec{e}_{p}=\frac{B_{p}}{B} \vec{e}_{\|}+\frac{B_{t}}{B} \vec{e}_{\perp}$, may be combined to give $\vec{e}_{\perp}=\frac{B_{t}}{B_{p}} \vec{e}_{\|}-\frac{B}{B_{p}} \vec{e}_{t}$, which converts Eq. 3 into following form:

$$
v_{\perp, \alpha} \vec{e}_{\perp}=\omega_{\alpha}(\Psi)\left(R \vec{e}_{t}-\frac{R B_{t}}{B} \vec{e}_{\|}\right)
$$

While not demonstrated here, it is known that $v_{\perp, \alpha} \vec{e}_{\perp}$ is not divergence-free. It is clear that the term $\propto R \vec{e}_{t}$ (rigid rotation) is divergence-free and thus is not responsible for the non-zero divergence of $v_{\perp, \alpha} \vec{e}_{\perp}$. However, the parallel term does lead to a non-zero divergence and thus requires a parallel flow with the same structure to compensate. Therefore, the parallel flows are described by

$$
v_{\|, \alpha} \vec{e}_{\|}=\omega_{\alpha}(\Psi) \frac{R B_{t}}{B} \vec{e}_{\|}+C B \vec{e}_{\|}
$$

where $\mathrm{C}$ is an undetermined constant allowing for a degree of freedom. A schematic visualization of the terms is presented in Fig. 1. The first term in Eq. 5 (in Fig.1 labelled $\vec{v}_{1, \|, \alpha}$ ) compensates for the non-zero divergence of the perpendicular velocity $v_{\perp, \alpha}$ and the second term describes an additional degree of freedom, as any parallel flow that is $\propto B$ is divergence free due to $\vec{\nabla} \cdot \vec{B}=0$. For $C=\frac{\hat{u}_{\alpha}}{\left\langle B^{2}\right\rangle}-\omega_{\alpha}(\Psi) \frac{R B_{t}}{\left\langle B^{2}\right\rangle}$, Eq. 5 may be rewritten in the following way

$$
v_{\|, \alpha} \vec{e}_{\|}=\omega_{\alpha}(\Psi) R B_{t}\left[\frac{1}{B}-\frac{B}{\left\langle B^{2}\right\rangle}\right] \vec{e}_{\|}+\hat{u}_{\alpha} \frac{B}{\left\langle B^{2}\right\rangle} \vec{e}_{\|}
$$

where $\left\langle B^{2}\right\rangle$ is the flux surface average of $B^{2}$ and $\hat{u}_{\alpha}$ a constant. This has the advantage that $\left\langle\vec{v}_{\|, \alpha} \vec{B}\right\rangle=\hat{u}_{\alpha}$, which is of interest for radial transport considerations. In the following the first term in Eq. 6 is referred to as the Pfirsch-Schlüter flows. This term is labelled $\vec{v}_{P S, \alpha}$ in Fig.1 resulting from $\vec{v}_{1, \|, \alpha}+\vec{v}_{2, \|, \alpha}$, where $\vec{v}_{2, \|, \alpha}=-\omega_{\alpha}(\Psi) R B_{t} \frac{B}{\left\langle B^{2}\right\rangle} \vec{e}_{\|}$.

Eqs. 4 and 5 may be combined to form one equation for $\vec{v}_{\alpha}$. This leads to a general form, which is used in Ref. [4] to investigate the flow structure on a flux surface and separates the 


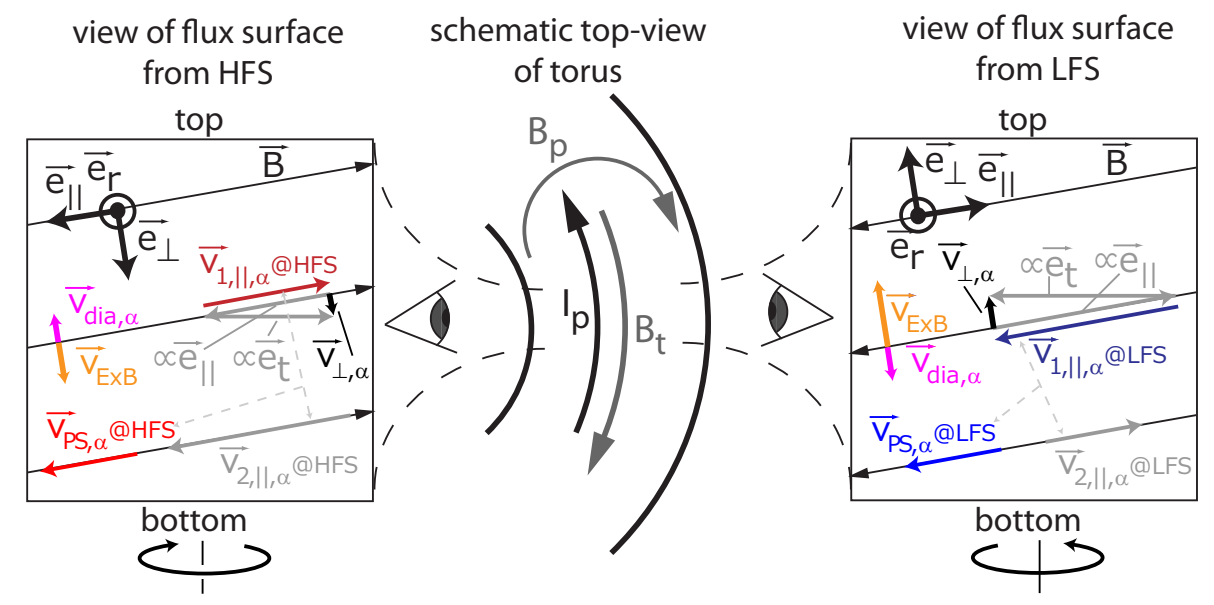

Figure 1. Directions of the various vectors for a typical case in ASDEX Upgrade on a flux surface at about $\rho_{\text {pol }}=0.975$. The perpendicular velocity $\vec{v}_{\perp, \alpha}$ of a species $\alpha$ is shown as the sum of the diamagnetic velocity $\vec{v}_{d i a}$ and $\vec{v}_{E x B}$ at the HFS and LFS. The $\vec{v}_{\perp, \alpha}$ is also represented by a sum of a toroidal and parallel vector (cf. Eq. 4). The compensating parallel term $\vec{v}_{1, \|, \alpha}$ is added to a term $\propto \vec{B}$, which is labelled $\vec{v}_{2, \|, \alpha}$ to obtain the Pfirsch-Schlüter flow $\vec{v}_{P S, \alpha}$ (cf. first term in Eq. 6). With respect to $\vec{B}, \vec{v}_{P S, \alpha}$ at the HFS has the opposite direction from $\vec{v}_{P S, \alpha}$ at the LFS. Please note that the length of the vectors is not exactly up to scale.

total flow into a rigid body rotation characterized by the angular frequency $\omega_{\alpha}$ and a parallel flow.

$$
\vec{v}_{\alpha}=\omega_{\alpha}(\Psi) R \vec{e}_{t}+C B \vec{e}_{\|}
$$

Equation 7 , can also be used to investigate the effect of a poloidal impurity density $\left(n_{\alpha}\right)$ variation on a flux surface. A poloidal redistribution is only provided by the second term in Eq. 7 and thus only this part of the flow is affected by a poloidal impurity density variation. As the particle flux is $\propto n_{\alpha}$ the second term needs to be $\propto 1 / n_{\alpha}$ in order to maintain the divergence-free structure. This was described in Ref. [4] and will be used later in this work. However, it should be noted that a poloidal change of the density will also influence the poloidal structure of the diamagnetic term in the radial force balance. Such a change could mean that the poloidal flow of the species or possibly even the electrostatic potential may have poloidal dependencies that are not expected.

It should also be noted that in the above the divergence of the radial transport was considered negligible. If the divergence of the radial flow is considerable, then it must be included in the continuity equation and the equations above require an additional term. For sections 2 to 7 , the radial transport and its divergence is ignored.

\section{Observation of the Flow Structure}

In the previous section, the cancellation of the parallel terms in Eq. 4 and Eq. 5 might give the impression that this structure, which is poloidally asymmetric, is not observable in experiment. However, in Eq. 4 the toroidal rotation closely matches the parallel term such 
that the perpendicular flow $v_{\perp, \alpha} \vec{e}_{\perp}$ is obtained. The projection onto the LOS of $v_{\perp, \alpha} \vec{e}_{\perp}$ is very small (s. below). At the same time, the parallel Pfirsch-Schlüter flow structure (Eq. 5) has no compensating term attached and thus is visible. If we compare numbers for the actual geometry of the diagnostics, the angle between magnetic field lines and the lines of sight (LOS) for the toroidal views is smaller than 10 degrees for all of the cases considered in this work. The maximum values in the perpendicular rotation, are in the range of $20 \mathrm{~km} / \mathrm{s}$, thus the projection on the (approximately) toroidal LOS $\left(<\sin 10^{\circ}\right)$ is smaller than $3.5 \mathrm{~km} / \mathrm{s}$. The parallel flow, for which the Pfirsch-Schlüter flow compensates, is expected to be in the range of $20-30 \mathrm{~km} / \mathrm{s}$ at the outer midplane and its projection onto the LOS $\left(>\cos 10^{\circ}\right)$ is virtually the full flow. As $20-30 \mathrm{~km} / \mathrm{s}$ are much larger than the upper bound for the observed perpendicular flow $(3.5 \mathrm{~km} / \mathrm{s})$, the Pfirsch-Schlüter flows dominate the toroidal flow measurement in comparison to the perpendicular flows.

For a direct comparison between theory and experiment, we can use the equality $\frac{\partial r}{\partial \Psi}=\frac{1}{R B_{p}}$ in order to rewrite the product of $\omega_{\alpha}$ and $R B_{t}$ (cf. first term on the right side in Eq. 6):

$$
\omega_{\alpha} R B_{t}=\left(-E_{r}+\frac{1}{Z_{\alpha} e n_{\alpha}} \frac{\partial p_{\alpha}}{\partial r}\right) \frac{B_{t}}{B_{p}}
$$

Due to the fact that $\omega_{\alpha}$ and $R B_{t}$ are constants on a flux surface the corresponding terms in Eq. 6 may be evaluated at the LFS and are valid for the full flux surface. Therefore, it is valid to rewrite Eq. 6 as

$$
\begin{aligned}
v_{\|, \alpha}(B) & =\left(-E_{r, L F S}+\left.\frac{1}{Z_{\alpha} e n_{\alpha}} \frac{\partial p_{\alpha}}{\partial r}\right|_{L F S}\right) \frac{B_{t, L F S}}{B_{p, L F S}}\left(\frac{1}{B}-\frac{B}{\left\langle B^{2}\right\rangle}\right)+\hat{u}_{\alpha} \frac{B}{\left\langle B^{2}\right\rangle} \\
& =v_{P S, \alpha}+\hat{u}_{\alpha} \frac{B}{\left\langle B^{2}\right\rangle}
\end{aligned}
$$

where $B_{t, L F S}, B_{p, L F S}, E_{r, L F S}$ and $\left.\frac{\partial p_{\alpha}}{\partial r}\right|_{L F S}$ denote the toroidal and poloidal magnetic field, the radial electric field and pressure gradient of the particle species $\alpha$ at one location on the LFS. This means that for the interpretation of the experimental data, the LFS measurements and the information about the magnetic equilibrium (from magnetic equilibrium reconstruction) can be used to obtain the Pfirsch-Schlüter flows $v_{P S, \alpha}$ on the full flux surface. Note that Eq. 9 is an implicit Definition for $v_{P S, \alpha}$ and exactly corresponds to $v_{P S, \alpha}$ that was introduced earlier, e.g. in Fig. 1.

In Fig. 2 the structure of the Pfirsch-Schlüter flow for a real geometry at the radius $\rho_{p o l}=0.975$ is indicated for the condition $-E_{r}+\frac{1}{Z_{\alpha} e n_{\alpha}} \frac{\partial p_{\alpha}}{\partial r}>0 \mathrm{kV} / \mathrm{m}$. This condition refers to the typical situation inside of the separatrix, where the radial electric field is negative and the absolute value is larger than the diamagnetic term of the impurities, which is also negative. Blue (red) regions indicate flows directed out of (into) the plane of the paper. The thickness of the region indicates the absolute size of the flow. It should be noted that the Pfirsch-Schlüter flows are a property of a flux surface, and thus the values of $E_{r}$ and the diamagnetic term of the species $\alpha$ at the considered radius are of importance. In Fig. 2, the measurement locations of the toroidal and poloidal (newly installed) rotation at the LFS are indicated along with the newly installed diagnostics for toroidal rotation at the HFS. The locations are well suited to see effects of Pfirsch-Schlüter flows.

In order to compare the flows as evaluated in Eq. 9 to the measurements, we evaluate $v_{P S, \alpha}$ in Eq. 9 from the experimental data at the LFS. We then use Eq. 9 to determine the constant $\hat{u}_{\alpha}$, as we know $v_{P S, \alpha}$ from the LFS measurements and we know $v_{\|, \alpha}$ at the LFS, which is to good accuracy equal to our measured toroidal velocity at the LFS (denoted as 


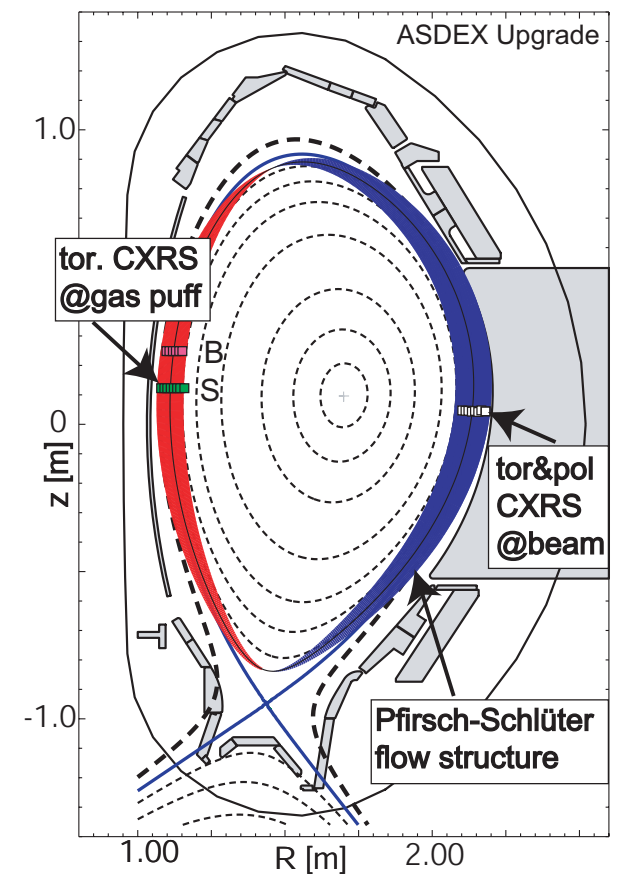

Figure 2. Structure of Pfirsch-Schlüter flows at $\rho_{\text {pol }}=0.975$ for $-E_{r}+\frac{1}{Z_{\alpha} e n_{\alpha}} \frac{\partial p_{\alpha}}{\partial r}>$ $0 \mathrm{kV} / \mathrm{m}$ and diagnostic locations for the beam and gas puff based CXRS systems.

$v_{\|, \alpha, L F S}^{e x p}$ in the following). After that we have all of the necessary ingredients to predict the parallel flow at the measurement locations on the HFS. Thus we are able to test the prediction by comparing to the measurement at the HFS (denoted as $v_{\|, \alpha, H F S}^{e x p}$ in the following). It should be noted that this procedure is equivalent to testing Eq. 7 with all three flow measurement (i.e. both toroidal and one poloidal flow measurement). The equivalence is maybe unclear, because for the test of Eq. 7 only flow measurements are required, while for the evaluation of the Pfirsch-Schlüter flows $E_{r}$ and the diamagnetic term is required. However, if $E_{r}$ is evaluated from the radial force balance of species $\alpha, E_{r}$ contains exactly the diamagnetic term, which is subtracted for the evaluation of the Pfirsch-Schlüter flows. In that case, Eq. 9 may be evaluated by knowing only the three flow measurements and the two procedures (i.e. exploiting Eq. 7 or Eq. 9) are also equivalent in terms of uncertainties. Note, that in this work Eq. 9 is also analyzed using the diamagnetic term of deuterium, while the flow measurements from impurities are used. This comparison is only possible using Eq. 9 and the uncertainties of the diamagnetic terms of the impurity species and of deuterium enter.

\section{Flow Diagnostic at HFS and LFS}

For the investigated discharges, the LFS and HFS rotation at the plasma edge are recorded with 2-4 ms time resolution. The rotation measurements at the LFS are performed via CXRS at a heating beam [6]. At the HFS, a D gas puff is applied which leads to CX reactions between the penetrating D gas cloud and the plasma impurities. With this method, information about rotation is obtained only in the outermost plasma region into which the neutrals penetrate, i.e a few $\mathrm{cm}$. A top-view of the edge diagnostics is provided by Fig. 3. Please note that 
the ' $S$ ' and 'B' LOS, which are visible in the poloidal view (Fig. 2), are exactly overlaid in the top-view. For the CXRS reaction between the thermal deuterium gas cloud and the fully

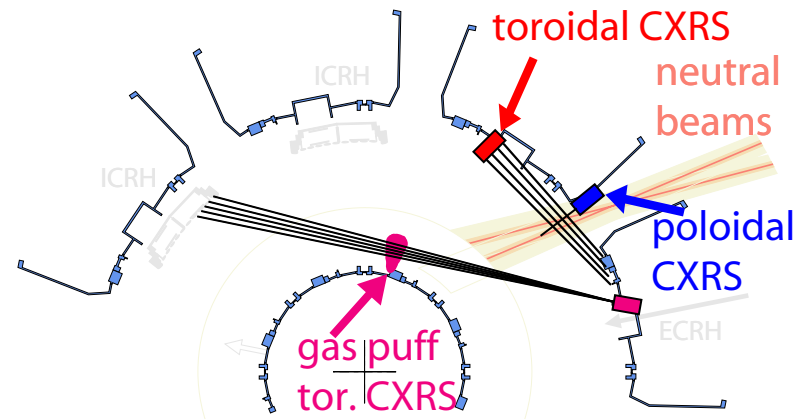

Figure 3. Top-view of the beam and gas puff based CXRS systems for the diagnosis of the LFS and HFS plasma edge. ' $B$ ' and ' $S$ ' stand for background and signal LOS, which are described in the text.

stripped impurity ions, the dominant electron donor is the population of the $n=2$ state in atomic deuterium. The cross section at thermal energies has only a weak energy dependence and thus the temperature dependence of the effective reaction rate coefficient is weak. Therefore, the Doppler shift of the CX spectral lines allows the direct determination of the impurity flow, while corrections due to cross section effects (cf. e.g. Ref. [7]) are negligible. However, the CX spectral line (active signal) is blended with passive emissions and special effort is required to isolate the active signal. Two toroidal arrays of LOS have been implemented at the HFS. One is looking directly at the gas puff and gathers the photons from the CX reactions (these LOS are labeled with 'S', for signal, in Fig. 2) along with background photons that are emitted at the plasma edge elsewhere on the paths of the LOS. The LOS of the second array run parallel to those, but miss the gas cloud (labeled 'B', for background, in Fig. 2) and thus provide equivalent spectra but without the CX signal such that the background signal in the active spectra (measured on the 'S' set of LOS) can be subtracted off. For the HFS measurement, a diagnostic gas puff is applied at a time of interest. A few milliseconds before the gas puff, the calibrations of the 'B' channels are refined, i.e. relative calibration factors are obtained with respect to the corresponding ' $S$ ' channels. During the gas puff the spectra from the 'B' channels are subtracted from those on the ' $S$ ' channels using the previously obtained calibration refinement. In order to provide good diagnostic coverage of the plasma edge, the plasma is swept by $2 \mathrm{~cm}$ such that the edge pedestal is slowly moved through the view of the LOS (cf. Ref. [6]).

It should be noted that in the following the flow measurements from the LFS and HFS $\left(v_{\|, \alpha, L F S}^{\exp }\right.$ and $\left.v_{\|, \alpha, H F S}^{\exp }\right)$ are denoted as toroidal rotation. However, the differences between parallel and toroidal velocities are very small due to the field line geometry. This systematic difference is typically smaller than the error bars which represent only the statistical uncertainty and hence, have been neglected. The wavelength calibration is obtained by inter-shot measurements using a Ne lamp and is better than $1 \mathrm{~km} / \mathrm{s}$. 


\section{Experiment}

The data from two discharge phases are presented in the following. In the first discharge, Ne-seeding leads to improved confinement and fast small ELMs [8], while in the other case a type-I ELMy H-mode [9] is investigated. Both plasma discharges are performed with a toroidal magnetic field of $2.5 \mathrm{~T}$, a plasma current of $1 \mathrm{MA}\left(q_{95} \approx 4.5\right)$, neutral beam heating of $5 \mathrm{MW}$, an additional $1.5 \mathrm{MW}$ of ECRH and a density of approx. $8 \cdot 10^{19} \mathrm{~m}^{-3}$. The H98(y,2)confinement-factor is 1.05 for the Ne-seeded case and 0.85 for the type-I ELMy H-mode.

At the LFS, the poloidal and toroidal CXRS measurements are used to obtain a profile of $E_{r}$. The alignment of the toroidal and poloidal CXRS data at the LFS is performed by a comparison of the $T_{i}$-profiles. The position of the separatrix is found by aligning the CXRS data to the electron temperature profiles for which the separatrix position is known from power balance analyses. The procedure is described in Refs. [10, 11, 12].

The HFS flow measurements $v_{\|, \alpha, H F S}^{\exp }$ are aligned to the LFS flow measurements $v_{\|, \alpha, L F S}^{\exp }$ (thus also to the $E_{r}$ data) using the $T_{i}$-profiles. The accuracy of the process is estimated to be better than 2-3 mm. In Fig. 4(a), an alignment of the HFS $T_{i}$-profile with respect to the LFS $T_{i}$-profile is presented. In Fig. 4(b), the toroidal rotation profiles for the same discharge are shown. As mentioned earlier the ELMs in this discharge are small, highfrequency perturbations, which have little effect on the edge plasma. The spectra obtained during an ELM crash have been removed from the analysis. The Ne-seeding allows the rotation of $\mathrm{Ne}^{10+}$ and $\mathrm{C}^{6+}$ to be derived from the same spectrum (CX-lines at $524.897 \mathrm{~nm}$ $(\mathrm{Ne})$ and $529.059 \mathrm{~nm}(\mathrm{C})$ ). The profile described by the red data (C) agrees within the uncertainties with that obtained from the green data $(\mathrm{Ne})$ and both data sets exhibit an asymmetric behavior with respect to the toroidal rotation measured at the LFS. For the latter, only $\mathrm{C}^{6+}$ data is presented for clarity; the Ne LFS data also agrees within the uncertainties of the measurements (cf. [3]). In Fig. 4(c), the poloidal rotation profile for $C^{6+}$ is depicted, which is important for the considerations on the Pfirsch-Schlüter flows. Please note that a strong poloidal rotation for the impurities is observed, as $E_{r}$ is governed by the main ions, i.e. deuterium, while the impurities have to fullfill their species-specific radial force balance. As the diamagnetic term of the impurities is much smaller than that for deuterium, a considerable perpendicular velocity and thus a strong poloidal velocity is required, while the poloidal velocity of deuterium may be quite small. A similar comparison of the parallel flow velocities is presented in Fig. 5, for the inter-ELM phase of a type-I ELMy H-mode. The presented data originate from the second half of the ELM cycle, excluding the ELM crash. The discharge was repeated to obtain data for $\mathrm{B}^{5+}(494.467 \mathrm{~nm})$ and for $\mathrm{C}^{6+}$. The shapes of the edge rotation profiles of the type-I and the Ne-seeding cases are similar. For the latter, the difference between the LFS and HFS rotation at about $\rho_{\text {pol }}=0.975$ is larger by about $10 \mathrm{~km} / \mathrm{s}$ than for the type-I case. For both cases, the LFS rotation increases and the HFS rotation decreases towards the separatrix, such that they cross inside of the separatrix. These basic observations are valid even if the profiles are shifted within the uncertainties of 2-3 $\mathrm{mm}$.

\section{Comparison of Experimental Flows with the Pfirsch-Schlüter Prediction}

In the following, the question of whether or not the observed flow velocities are consistent with a divergence free flow structure on a flux surface will be addressed. This comparison is the most important result of the present work. For this, the $E_{r}$ measurement, i.e. the toroidal and poloidal rotation measurement, performed at the LFS are used. For the Ne-seeding case, $E_{r}$ (black) is presented in Fig. 6(a). In Fig. 6(b) the Pfirsch-Schlüter-term $v_{P S, \alpha}$ and the $\hat{u}_{\alpha}$ 

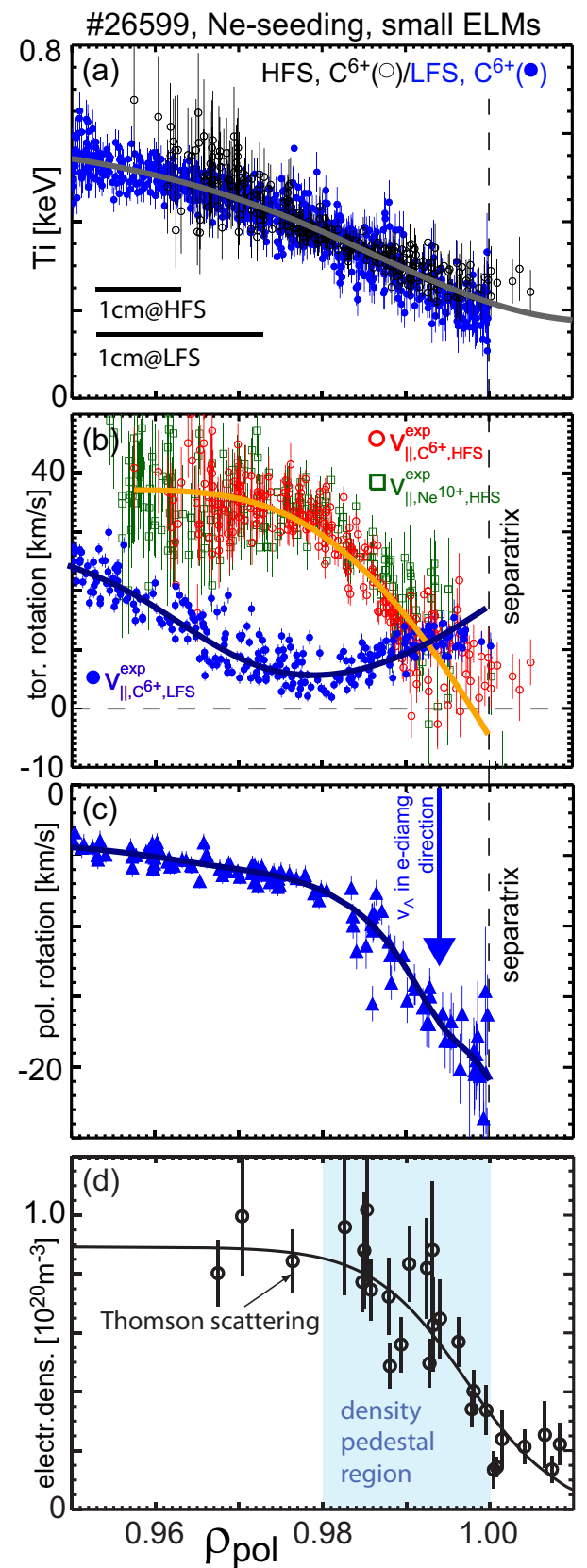

Figure 4. (a) HFS/LFS measurements of $T_{i}$ to accomplish the alignment of the rotation profiles. (b) Toroidal/parallel rotation at HFS/LFS. (c) Poloidal rotation of $\mathrm{C}^{6+}$ as measured via an independent, poloidal viewing geometry. (d) The electron density as measured by Thomson scattering is provided as an independent identification of the density pedestal region. The lines are spline fits to the data. 


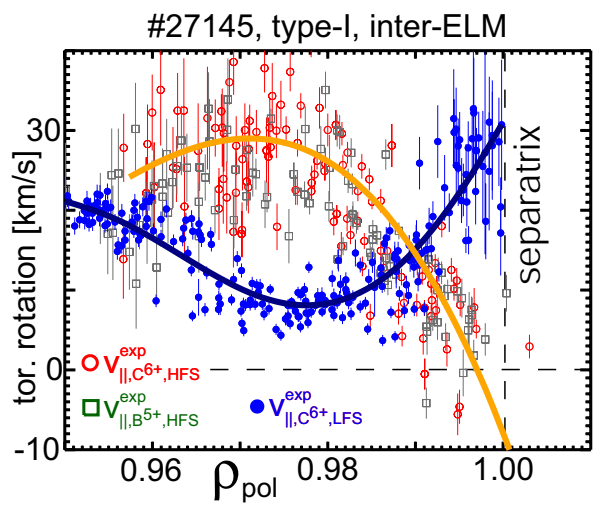

Figure 5. Toroidal/parallel rotation at HFS/LFS for $C^{6+}$ in $\# 27148$ and toroidal/parallel rotation at HFS for $B^{5+}$ in \#27145.

term at the LFS as derived from the measurements are presented. In order to predict $v_{\|, \alpha}$ (cf. Eq. 9) at the HFS, the measured parallel flow $v_{\|, \alpha, L F S}^{\exp }$ at the LFS is compared to $v_{\|, \alpha}$ at the LFS from Eq. 9 and the difference is attributed to $\hat{u}_{\alpha} \frac{B_{L F S}}{\left\langle B^{2}\right\rangle}$, which determines the constant $\hat{u}_{\alpha}$. Thus $\hat{u}_{\alpha} \frac{B}{\left\langle B^{2}\right\rangle}$ at the HFS is known using the local B. This allows for the evaluation of $v_{\|, \alpha}$ at the HFS (Eq. 9). In Fig. 6(c), the predicted HFS rotation profile (green) is plotted and compared to the measurement $v_{\|, C^{6+} / N e^{10+}, H F S}^{\exp }$ (i.e. the spline fit (blue) to the experimental data), which corresponds to that in Fig. 4(b). The green error bars indicate the propagated error due to an uncertainty of $\pm 5 \mathrm{~km} / \mathrm{s}$ in $v_{\|, C^{6+} / N e^{10+}, L F S}^{e x p}$ (at the LFS). The uncertainty $\pm 5 \mathrm{~km} / \mathrm{s}$ corresponds approx. to the scatter of the data around the spline fit. The measurement agrees with the prediction up to a radius of about $\rho_{\text {pol }}=0.98$. Outside of $\rho_{\text {pol }}=0.98$ strong discrepancies in the values of the flow velocity and also in its radial gradient appear. While the measured, parallel flow velocity decreases towards the separatrix the prediction increases. At the separatrix the parallel flow velocity is measured to be about $0 \mathrm{~km} / \mathrm{s}$, while the prediction is in the range of $70 \mathrm{~km} / \mathrm{s}$. For the inter-ELM phase in the type-I ELM case (Fig. 6(d), 6(e) and 6(f)) similar observations are made. Due to the lower scatter in the data, the Ne-seeding case is better suited to argue about the HFS flows, however, as the type-I ELMy H-mode is a more common scenario it is presented along with the Ne-seeding data. When looking at Fig. 6, a consistent match of the LFS and HFS velocities would only be possible, if at the pedestal region $E_{r}$ was more closely matched by the diamagnetic term and, as a consequence, the calculated $v_{P S, \alpha}$ would be closer to 0 ; this is the case for deuterium.

Thus the idea is to investigate the following hypothesis: The parallel flows of the impurities are more strongly influenced by the parallel deuterium flow than neoclassical theory accounts for. This might lead to a non-zero divergence of the impurities. The latter implies a systematic, poloidal density variation, a poloidally asymmetric radial transport (i.e. radial transport with non-zero divergence) or both. Note that this hypothesis would be in disagreement with the neoclassical picture, where the impurity flows exhibit no divergence and the difference in the Pfirsch-Schlüter flows is closely connected to neoclassical radial transport. However, indications that the impurities are dragged along in the parallel deuterium flows have been observed in Ref. [3]. If this hypothesis holds, the measured parallel impurity flows would give us the parallel deuterium flows and we can test, whether the deuterium flows agree with Eq.9, i.e. are divergence-free. In order to test the hypothesis, we evaluate 

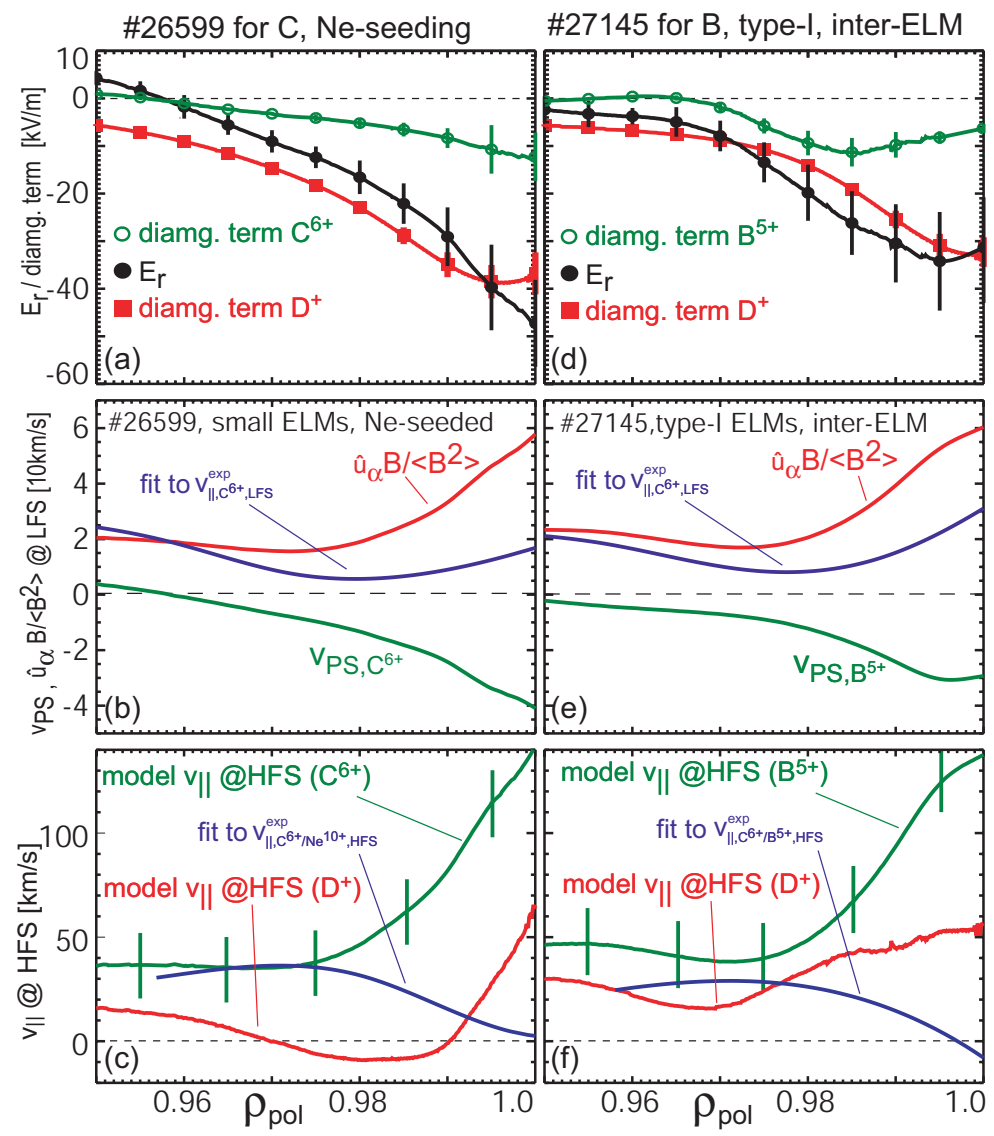

Figure 6. (a) $E_{r}$ and diamagnetic terms of $D^{+}, C^{6+}$ for \#26599. (b) The two terms in Eq. 9 are presented as evaluated at the LFS measurement position. $v_{P S}$ (green) is determined from $E_{r}$ and diamagnetic term (cf part (a)), $\hat{u}_{\alpha}$ (red) is determined such that the measurement (blue) is obtained by Eq. 9. (c) The fit to the measurements $v_{\|, C^{6+} / N e^{10+}, H F S}^{\text {exp }}$ (blue) is compared to $v_{\|}$evaluated by Eq.9 at the HFS (green). The red curve corresponds to the model curve, when $v_{P S}$ is evaluated with the diamagnetic term of deuterium. Further explanation is in the text. The parts (d),(e) and(f) are the corresponding figures for discharge \#27145.

$v_{P S, \alpha}$ for deuterium using Eq. 9, under the assumption that the LFS flows of deuterium and impurity flows are equal. For deuterium the match between $E_{r}$ and the diamagnetic term (cf. Fig. 6(a)) is very close and, thus the agreement of the HFS flow prediction (red in Fig. 6(c)) and the measurement $v_{\|, C^{6+} / N e^{10+}, H F S}^{\exp }$ (performed on the impurity) is indeed slightly better between $\rho_{\text {pol }}=0.98$ and the separatrix. However, the description is still not satisfactory and does not seem to catch all of the important physics.

On top of that, inside of $\rho_{\text {pol }}=0.98$ the measurement of the $\mathrm{C}^{6+} / \mathrm{Ne}^{10+}$-flows at the HFS are clearly different from the expectations for D flows. For the type-I ELM case (cf. Fig. 6(f)) the measured flows are not described by the the calculated parallel flows, too. Both studied cases show that the D-flow and the Ne/C/B-flows are different, which also means that there should be a difference between the flows of the various impurities. This difference is estimated to be relatively small compared to the scatter in the measurement and, therefore, 
it is not visible. Future investigations will try to better quantify that difference.

The discrepancy of the flow prediction and the measured flows suggests that one of the assumptions made for deriving Eq. 9 is violated. The assumptions are that the divergence of radial transport is negligible compared to parallel transport and that the impurity density is constant on a flux surface. In principle, there is a close interconnection between the two, because the divergence of radial transport will most probably influence the poloidal asymmetry of the impurity density and the flow structure on a flux surface. For negligible divergence of the radial transport, an impurity density asymmetry is sufficient to alter the flow structure. For that case the impurity flux density, i.e. the flow velocities times the local impurity density, need to be divergence free, as demanded by the continuity equation.

\section{Interpretation of the Discrepancies as an Impurity Density Asymmetry}

In this section we assume that the divergence of radial transport $\left(n_{\alpha} v_{r} \vec{e}_{r}\right)$ is negligible and we derive a density asymmetry which, in combination with our measured flows, results in a divergence-free situation on each flux surface. We follow the concept presented in Ref. [4] to derive that asymmetry, which means that we alter Eq. 7 to accommodate a poloidal impurity density variation. As the toroidal term in Eq. 7 does not poloidally redistribute the particles, we need to introduce the impurity asymmetry only in the second term. We get

$$
\vec{v}_{\alpha}=\omega_{\alpha}(\Psi) R \vec{e}_{t}+\frac{\tilde{C}}{n_{\alpha}} B \vec{e}_{\|}
$$

The first part in Eq. 10 corresponds to the toroidal component of the perpendicular velocity. Thus using the local field line inclination angle at the LFS $\delta_{L F S}$ the expression may be replaced by $\frac{v_{\perp, \alpha, L F S}^{\exp }}{\sin \delta_{L F S}} \frac{R}{R_{L F S}}$, where $v_{\perp, \alpha, L F S}^{\exp }$ is the perpendicular velocity at the LFS, which is determined mostly by the poloidal velocity, and $R_{L F S}$ is the the large radius at the same position on the LFS. The term $\frac{R}{R_{L F S}}$ is necessary, to maintain the validity of the replacement expression for the full flux surface. The simplification that the toroidal and the parallel direction are indistinguishable for the used viewing geometries at LFS and HFS has two consequences. First, the measured (toroidally) projections of $\vec{v}_{\alpha}$ at LFS and HFS, i.e. $v_{\|, \alpha, L F S}^{e x p}$ and $v_{\|, \alpha, H F S}^{e x p}$, are not sensitive to the perpendicular nor to the poloidal part of $\vec{v}_{\alpha}$. Second, the measurement $v_{\|, \alpha, L F S}^{e x p}$ at the LFS, may be compared to

$$
v_{\|, \alpha} \approx \frac{v_{\perp, \alpha, L F S}^{e x p}}{\sin \delta_{L F S}} \frac{R}{R_{L F S}}+\frac{\tilde{C}}{n_{\alpha}} B
$$

(corresponding to Eq. 10) when evaluated at the LFS. Note that the toroidal viewing geometry cannot distinguish between the first and second term in Eq. 11, but the additional poloidal rotation measurement at the LFS allows for the determination of $v_{\perp, \alpha, L F S}^{e x p}$ and thus of the first term. Note that on the right hand side the first term ought to be multiplied by $\cos \epsilon_{1}$ and the second term by $\cos \epsilon_{2}$, where $\epsilon_{1}$ is the angle between LOS and $\vec{e}_{t}$ and $\epsilon_{2}$ is the angle between the LOS and $\vec{e}_{\|}$. This has been omitted for simplicity and the introduced error is negligible, because the cosine of both angles is greater than 0.98 .

The second term on the right hand side of Eq. 11 (i.e. $\frac{\tilde{C}}{n_{\alpha}} B$ ) is determined by setting $v_{\|, \alpha}=v_{\|, \alpha, L F S}^{e x p}$. Thus it is equal to the difference between the measurement of $v_{\|, \alpha, L F S}^{e x p}$, and $\frac{v_{\perp, \alpha, L F S}^{e x p}}{\sin \delta_{L F S}}$. In order to obtain $v_{\|, \alpha}$ on the full flux surface, this term must be scaled with $\frac{B}{B_{L F S}}$ and $\frac{n_{\alpha, L F S}}{n_{\alpha}}$, where $n_{\alpha, L F S}$ is the impurity density at the LFS position. Thus $\tilde{C}=$ 
$\frac{n_{\alpha, L F S}}{B_{L F S}}\left[v_{\|, \alpha, L F S}^{\exp }-\frac{v_{\perp, \alpha, L F S}^{e x p}}{\sin \alpha_{L F S}}\right]$. Using the considerations above, Eq. 11 may be rewritten to give

$$
v_{\|, \alpha, H F S}^{\exp } \approx \frac{v_{\perp, \alpha, L F S}^{\exp }}{\sin \delta_{L F S}} \frac{R_{H F S}}{R_{L F S}}+\frac{1}{F_{\frac{H F S}{L F S}}}\left[v_{\|, \alpha, L F S}^{\exp }-\frac{v_{\perp, \alpha, L F S}^{\exp }}{\sin \delta_{L F S}}\right] \frac{B_{H F S}}{B_{L F S}}
$$

where $F_{\frac{H F S}{L F S}}=\frac{n_{\alpha, H F S}}{n_{\alpha, L F S}}$ is the impurity asymmetry factor between the LFS and HFS impurity density. Thus $F_{\frac{H F S}{L F S}}$ may be determined by:

$$
F_{\frac{H F S}{L F S}} \approx \frac{\left[v_{\|, \alpha, L F S}^{e x p}-\frac{v_{\perp, \alpha, L F S}^{e x p}}{\sin \delta_{L F S}}\right] \frac{B_{H F S}}{B_{L F S}}}{v_{\|, \alpha, H F S}^{e x p}-\frac{v_{\perp, \alpha, L F S}^{e x p}}{\sin \delta_{L F S}} \frac{R_{H F S}}{R_{L F S}}}
$$

The evaluation of Eq. 13 is presented in Fig. 7 for both investigated discharges. The discrepancies between the predicted and measured toroidal velocities on the HFS (cf. Fig. 6) translate into impurity density asymmetries factors of 3.9 and 6.5 at the separatrix. For the presented impurity asymmetries a perfect match of all measured flow velocities is obtained. Inside of the pedestal-top (of the electron density), the observed velocities do not require a density asymmetry and the presented curves approach 1 . The dashed lines indicate the error interval due to an uncertainty of $5 \mathrm{~km} / \mathrm{s}$ in the toroidal velocity measurements and $1 \mathrm{~km} / \mathrm{s}$ in the poloidal velocity measurements. We consider the postulated asymmetry in impurity density quite large and thus emphasize that an independent test of that result is necessary.
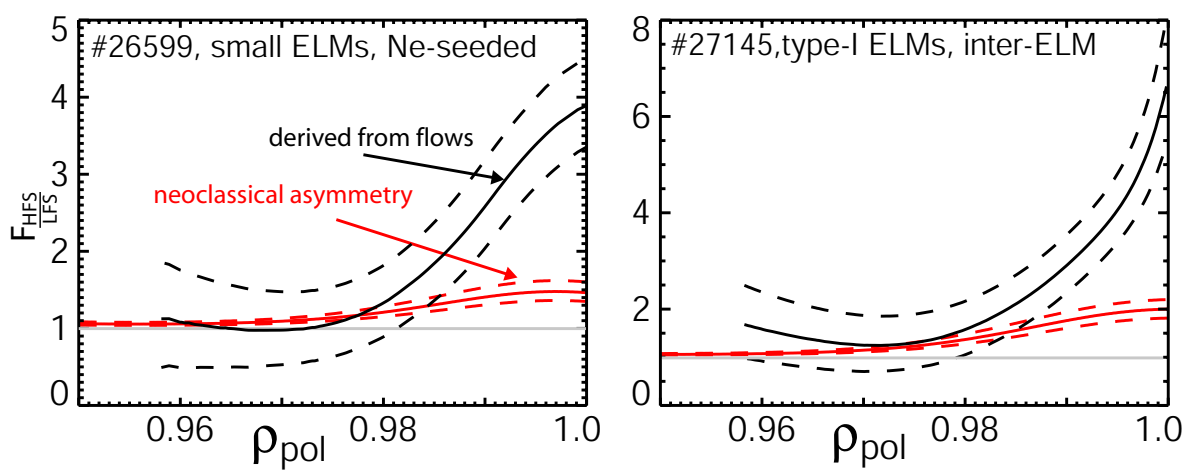

Figure 7. The postulated impurity density asymmetries, as derived from the flow measurements on LFS and HFS (black), is compared to the asymmetry as derived in Ref. [4] using the neoclassical scheme from Ref. [13] (red). The dashed lines indicate the uncertainty intervals.

In Ref. [4], it was found that the impurity density asymmetry cannot be described by a neoclassical scheme (cf. Ref. [13]), which allows for impurity asymmetries in regions with strong gradients in ion densities and ion temperatures. The same result is found for the analyzed discharges in the present work. Using the same scheme as in Refs. [4, 13], the experimental gradient lengths of ion densities (derived from the measured electron density profiles) and ion temperatures are used to evaluate the neoclassical asymmetry. The result is presented in red in Fig. 7. The uncertainty in the red curve is obtained by assuming a $40 \%$ uncertainty in the ratio of the ion density and ion temperature gradient lengths. The largest neoclassical asymmetries are found close to the separatrix, but they are less than a factor of two for both cases. 
Independently of that finding, a clear correlation between the magnitude of impurity asymmetry and the magnitude of poloidal velocity of the impurities is found in Ref. [4]. In the present work, the magnitude of the density asymmetry correlates with the distance to the separatrix, which is equivalent with the magnitude of the poloidal rotation (cf. Fig. 4(c)).

A quantitative evaluation of the impurity densities at LFS and HFS by CXRS is desireable acting as an independent confirmation of the large asymmetry postulated from the flow measurements. However, this requires a quite accurate, quantitative model for the penetration and excitation of the gas puff, which is not available at present. Further investigations into that direction are planned.

\section{Considerations on Radial Transport and Its Divergence}

All considerations in this chapter investigate the possibility of a non-negligible influence of poloidal asymmetric transport, i.e. radial transport with a considerable divergence of $n_{\alpha} v_{r} \vec{e}_{r}$, where $v_{r}$ is the radial flow velocity.

\subsection{Time Scales}

In Fig. 8, typical time scales of the processes that might influence the flow equilibrium on a flux surface are presented versus normalized radius. For the following considerations the Neseeded case was chosen. The processes for which the time scales are estimated are radial transport, redistribution on a flux surface along the field lines with ion sound speed and redistribution on a flux surface perpendicular to the field lines according to the perpendicular flows. The idea of the comparison is that radial transport could interfere with the flow equilibrium on a flux surface, by transporting impurities from an erosion location to the flux surface of interest. This would mean that the divergence of $n_{\alpha} v_{r} \vec{e}_{r}$ is non-zero and could explain the flow structure, a poloidal asymmetry in the impurity density or both. The parallel flows on a flux surface will try to equilibrate these asymmetries and if they manage, the divergence of the radial transport might be considered small with respect to the parallel flows, which is the assumption that we used to derive the 2D flow structure in section 2. The role of the perpendicular velocity is not quite clear, as on the one hand it provides a poloidal redistribution but on the other hand it drives up-down asymmetries that lead to the parallel Pfirsch-Schlüter flows. This ambiguity will be discussed below.

For the radial transport, the time scale is assessed in two ways: For radial particle transport, the typical time scale $\tau_{r, n}$, may be estimated by $\tau_{r, n}=L_{\alpha}^{2} / D$ (grey), where $L_{\alpha}$ is the gradient length of the impurity density $\left(\frac{1}{L_{\alpha}}=\frac{\partial n_{\alpha}}{\partial r} \frac{1}{n_{\alpha}}\right)$ and $D$ is the local diffusion coefficient. In Ref. [12] the radial transport of impurities on AUG has been investigated and for carbon $D \approx 0.2 \mathrm{~m}^{2} / \mathrm{s}$ was found in agreement with neoclassical values. Thus $D=0.2 \mathrm{~m}^{2} / \mathrm{s}$ is used here. For simplicity, we ignore the inward convection, which is also determined in Ref. [12].

The time scale for the radial momentum transport is not as easily calculated, because gradient lengths are not well defined for rotation velocities. At the same time it is known that $E_{r}$ is strongly changing within a few millimeters around the separatrix, as a few millimeters inside of the separatrix $E_{r}$ exhibits a global minimum at negative values for $E_{r}$, while in the SOL $E_{r}$ is positive. In order to accommodate these boundary condition for the estimate, a diffusion process is assumed for the momentum transport (using $\chi=D, \chi$ being the momentum diffusivity), where instead of $L_{\alpha}$ the distance to the separatrix $(\Delta x)$ is used. The associated typical time scale is denoted as $\tau_{r, p}=\Delta x^{2} / \chi$ (black). For the parallel time scale, the connection length along the magnetic field lines between LFS and HFS is evaluated and 


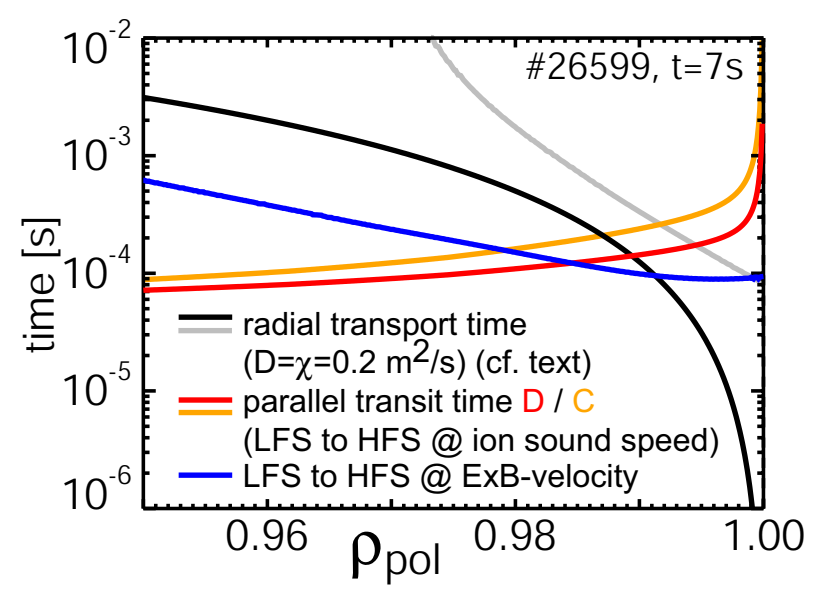

Figure 8. Comparison of radial, poloidal and parallel time scales. For radial regions where the parallel time scales are short compared to radial time scales the flows within a flux surface are expected to form a divergence free, $2 \mathrm{D}$ flow equilibrium. For radii greater than $\rho_{\text {pol }}=0.98$ the assumption is not quite valid.

divided by the ion sound speed. The latter is evaluated for both $\mathrm{D}$ (red) and for C (orange). The differences, however, are small, because for the impurity a correction due to the drag of electrons is included, which accelerates the impurities ions. For the perpendicular time scale (blue), the poloidal connection length via a flux surface between HFS and LFS is calculated (i.e. of the order $2.0 \mathrm{~m}$ ) and divided by the $E \times B$ velocity.

When approaching the separatrix (i.e. outside of $\rho_{\text {pol }}=0.98$ ), the time scale of radial transport to/from the SOL (black, grey) becomes comparable to the parallel transit time of $\mathrm{D}$ and the $\mathrm{C}$ ions. The radial density transport (grey, $\tau_{r, n}$ ) is estimated to be slower than the momentum transport (black, $\tau_{r, p}$ ), which might be an artefact of the way the time scales were estimated. Regardless, both estimates indicate that in the pedestal region radial transport is comparable to parallel redistribution and poloidal asymmetries of particle and momentum sources might be carried to these radial positions. Thus it is understandable that the flow structure described by Eq. 9 is violated outside of $\rho_{\text {pol }}=0.98$ as particle and/or momentum transport from neighboring flux surfaces might become relevant via radial transport. This could either transport poloidal asymmetries to the flux surface of interest or influence the flow structure via a non-zero divergence. The importance of radial transport outside of $\rho_{\text {pol }}=0.98$ is also acknowledged in Refs. [14, 15], where an influence of the scrape-off layer flows on the pedestal flows via viscosity is postulated. The respective B2 modeling in Refs. [14, 15] exhibits similar structures of parallel flows as measured in Ref. [3].

Additionally, the perpendicular velocity is large at the pedestal such that the drift time from LFS to HFS (blue) becomes comparable or even faster than the radial (black, grey) and the parallel transport time (red, orange). For a steady state situation this might not be important, because an equilibrium will form including consistent $E_{r}$ and Pfirsch-Schlüter flows. However, in combination with radial transport and density asymmetries the obtained effect is rather unclear. 


\subsection{Non-Zero Divergence due to Poloidally Asymmetric Radial Transport of Impurities}

Poloidal Asymmetries in transport (i.e. particle fluxes) exist naturally in turbulent and collisional transport models. At the plasma edge, these asymmetries could lead to density asymmetries because of the comparable time scales of radial and parallel transport. These effects could be taken account of in the presented equations by including a source and a sink term of the particle fluxes on a flux surface, which corresponds to the transport asymmetries.

Even for poloidally symmetric radial flow velocities, the particle fluxes could be asymmetric, if there are poloidally asymmetric recycling/erosion pattern of Deuterium and Impurities. These patterns could be propagated towards the confinement region of the plasma via radial transport, if parallel transport is sufficiently small.

In order to disentangle these possibilities more information, e.g. a poloidally resolved impurity density measurement, is required.

\subsection{Non-Zero Divergence due to an Ionization Source}

A polloidally localized ionization source, like the ionization source at the X-point region just inside of the separatrix leads to a non-zero divergence of the flows on the flux surface. Such a source leads to a parallel flow that redistributes particles on a flux surface, while the particles are also transported radially. An X-point ionization source could support a flow that for the used geometry is in co-direction on the LFS and in counter-direction on the HFS.

The following rough estimate assumes that the impurity content of the plasma is maintained by an impurity source at the edge. The magnitude of the source is quantified by assuming that the impurity confinement time is equal to the energy confinement time. It is assumed that $50 \%$ of the particles enter the plasma at the X-point and that $50 \%$ of these particles are transported along field lines to the upper half of the plasma. Even if that X-point source is a pure deuterium source the impurities will see effects of these flows via friction, which will lead to asymmetries in the impurity density.

The estimate results in parallel flow velocities of several tens of $\mathrm{km} / \mathrm{s}$. However, the details of such flows depend not only on the total X-point source, but also on the radial shape of that X-point source and on the poloidal structure of the radial transport in the main plasma. Thus the estimate only motivates further investigations and cannot be considered meaningful for quantitatively understanding the observations.

\section{Summary}

The flow structure in the H-mode edge of ASDEX Upgrade has been investigated. Three independent measurements allowed for the investigation of the flows on the flux surfaces from the separatrix to the pedestal-top. At the outboard midplane, i.e. low-field side (LFS), charge-exchange recombination spectroscopy (CXRS) measurements were performed on a heating beam and provided parallel and poloidal impurity flow velocities. At the inboard midplane, i.e. high-field side (HFS), a CXRS measurement has been installed, which relies on the interaction of a localized D gas puff and the impurities of the plasma. This diagnostic provides the parallel impurity flow velocities at the inboard midplane. The measurements have been performed in a Ne-seeded phase, in which the edge localized modes (ELMs) cause negligible perturbations and in the inter-ELM phase of a type-I ELMy H-mode. The results/conclusions from both cases are similar. The parallel flows at the LFS and HFS have strongly antisymmetric features. The parallel flow at the LFS exhibits a local minimum at the pedestal-top of the electron density, while at the HFS a local maximum is observed at 
approximately the same normalized radius. At this radius the HFS flows are larger than the LFS flows. From the pedestal-top towards the separatrix the LFS flow increases, while the HFS flow decreases, such that they become equal between the pedestal-top and the separatrix. At the separatrix, the LFS flow is larger than the HFS flow by a few tens of $\mathrm{km} / \mathrm{s}$.

The measurements are compared to a basic model of divergence-free flows on each flux surface. This theory neglects impurity density variations on a flux surface and the divergence of radial transport. The measurements are found to be consistent with the model inside of the pedestal-top of the electron density, i.e. inside of $\rho_{\text {pol }}=0.98$. However, at the pedestal itself it is inconsistent. Both is considered the main result of the present work. Further considerations are explored to identify which of the assumptions might be inapplicable at the pedestal.

First, the theoretical scheme is extended to allow for a poloidal asymmetry in the impurity density. This allowed a consistent description of the measurement, but requires a factor of 3.9/6.5 (Ne-seeded/type-I ELMy) higher densities at the HFS compared to the LFS at the separatrix. This postulated factor decreases for flux surfaces closer to the pedestal-top and is equal to about 1 (i.e. no asymmetry) at the pedestal-top. Due to the impressive magnitude of the asymmetry at the pedestal an independent confirmation by quantitative CXRS is desireable and planned.

Second, the importance of radial transport and its divergence is investigated. When approaching the separatrix, the time scale of radial transport becomes comparable to the time scales of transport connecting different locations on the same flux surface. This is the case for parallel transport, where particles can equilibrate perturbations with ion sound speed, as well as for perpendicular $E \times B$ convection. It is concluded that an asymmetric source of impurities could be the reason for an impurity density asymmetry and, thus for the observed flow structure. Independently, an asymmetric deuterium source or radial momentum transport could also lead to a similar result. For this mechanism it is in question whether the impurities exhibit a X-point source or whether the X-point ionization source of deuterium drives deuterium flows which drag impurities along. Both mechanisms will result in impurity density asymmetries. Further investigations are needed to fully understand the observed flow velocities.

\section{References}

[1] F. Wagner, ASDEX team, and NI team, Basic Physical Processes of Toroidal Fusion Plasmas (PUBLISHER, Varenna, 1985), Vol. I, pp. 65-85, eUR 10418 EN.

[2] A. Herrmann and O. Gruber, Fusion Science and Technology 44, 569 (2003).

[3] T. Pütterich et al., Physical Review Letters 102, 025001 (2009).

[4] K. D. Marr et al., Plasma Physics and Controlled Fusion 52, 055010 (2010).

[5] S. P. Hirshman and D. J. Sigmar, Nuclear Fusion 21, 1079 (1981).

[6] E. Viezzer et al., Review of Scientific Instruments -, (2012), to be submitted soon.

[7] R. J. Fonck, D. S. Darrow, and K. P. Jaehnig, Physical Review A 29, 3288 (1984).

[8] J. Schweinzer et al., Nuclear Fusion 51, 113003 (2011).

[9] W. Suttrop, Plasma Physics and Controlled Fusion 42, A1 (2000).

[10] E. Wolfrum et al., Plasma Physics and Controlled Fusion 51, 124057 (9pp) (2009).

[11] J. Neuhauser et al., Plasma Physics and Controlled Fusion 44, 855 (2002).

[12] T. Pütterich et al., Journal of Nuclear Materials 415, S334 (2011).

[13] T. Fulop and P. Helander, Physics of Plasmas 8, 3305 (2001).

[14] V. Rozhansky et al., Journal of Nuclear Materials 415, S593 (2011).

[15] V. Rozhansky et al., Journal of Nuclear Materials 363-365, 664 (2007). 